\title{
Sur la technique des biopsies musculaires
}

\section{Un retour historique}

Michel Fardeau

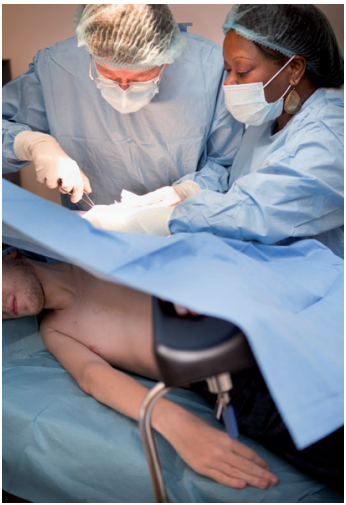

Professeur honoraire au CNAM, Fondateur de la Société Française de Myologie, Paris, France.

Martin Charcot. Ce dernier fit également l'examen anatomique de la moelle épinière et des nerfs périphériques de l'un de ses patients, pour en constater l'intégrité. Ce qui permit à Duchenne d'en affirmer l'origine primitivement musculaire.

\section{Les études post-mortem}

Son exemple fut peu suivi, et les études anatomo-pathologiques du tissu musculaire restèrent limitées au matériel recueilli post-mortem (Heller, Eulenburg et Connheim en Allemagne). Un autre instrument fut introduit plus tard, en 1943, par Shank et Hoagland, mais sans plus de succès. Jusqu'aux années 1950, l'essentiel des données fut tiré du matériel d'autopsie. D'importantes séries furent analysées (R. Adams, D. Denny-Brown, C. Pearson [2]), avec de longs développements concernant la sélection des territoires musculaires examinés, les techniques de fixation (solutions de Zenker, de Bouin, formaldéhyde à $20 \%$ ), et de coloration (hématoxyline-éosine, trichrome de Masson pour l'essentiel). Des colorations au chlorure d'or, selon Cajal, aux sels d'Argent (GrosBielchowsky) pouvaient être utilisées pour visualiser les nerfs intramusculaires et leurs terminaisons. L'analyse des différents artéfacts que les manipulations de prélèvement et de fixation entraînaient (en particulier les «bandes de contraction » décrites par Nageotte) fut faite avec soin. Cependant, les techniques ne permettaient guère d'aller au-delà d'une distinction assez grossière entre les atrophies par dénervation - dans lesquelles les fibres atrophiques étaient groupées en fascicules - et les atteintes cachectiques ou par immobilisation dans lesquelles les fibres présentaient une «atrophie simple», sans altération de leur structure myofibrillaire, ou les atteintes dystrophiques, dans lesquelles

Vignette (Photo @ AFM-Téléthon/Christophe Hargoues) 


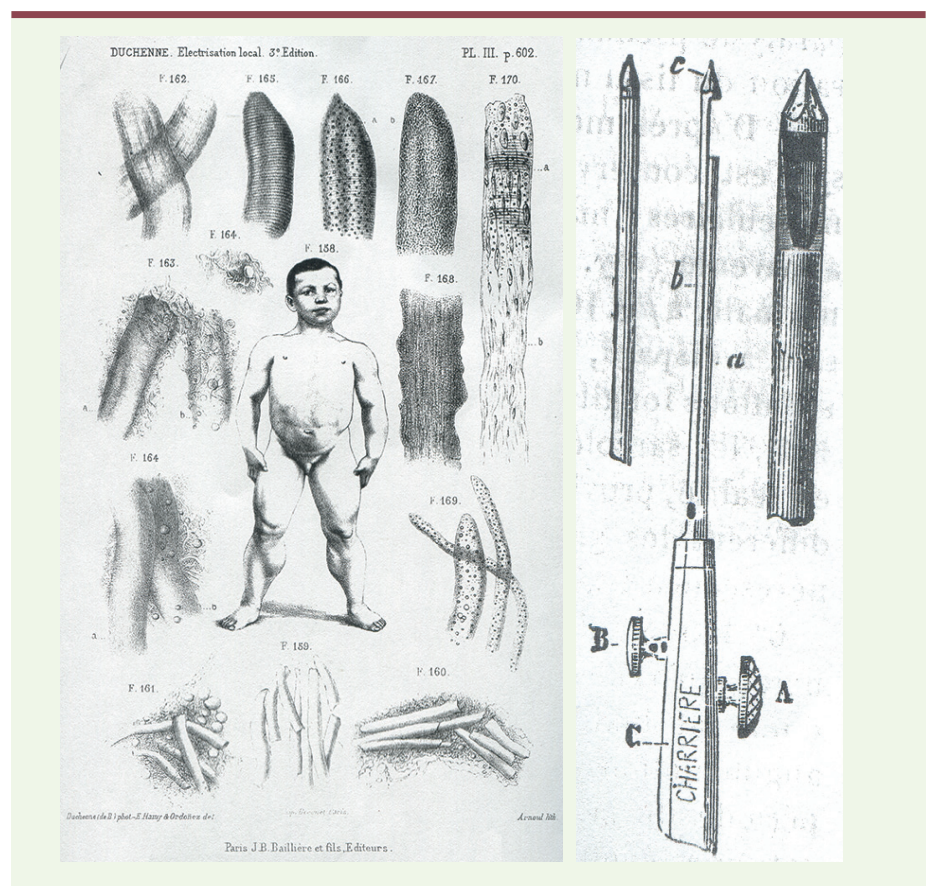

prédominaient des lésions dégénératives segmentaires (hyalines, nécrosantes, etc.), distribuées de façon aléatoire, et accompagnées d'une «surcharge » fibro-adipeuse plus ou moins intense. Les atteintes inflammatoires, groupées sous le terme de «dermatomyosites » étaient identifiées par la présence de nombreux aspects «dégénératifs » ou « régénératifs » et la présence d'une infiltration inflammatoire interstitielle plus ou moins marquée. Tel était l'état de la pathologie musculaire dans les laboratoires de neuropathologie jusqu'à la fin des années 1950.

\section{Détour par les études de l'innervation motrice terminale}

1943 avait vu la découverte de la structure des zones de jonction entre neurite moteur et fibre musculaire, avec la description précise des plaques motrices et de l'appareil sous- neural par R. Couteaux. Grâce à la collaboration entre ce dernier et $D$. Nachmansohn, avait été vue la concentration de cholinestérase dans les zones de contact nerfmuscle. Les premières images obtenues en microscopie électronique avaient confirmé, sur des matériels variés, le bien-fondé de ces observations. En 1949, Koëlle avait réussi à visualiser cette activité cholinestérasique dans les zones de contact. La méthode décrite par Koëlle et Friedenwald avait été affinée par Couteaux et Taxi en 1952 [3].

Comme l'une des grandes questions que les examens anatomo-pathologiques ne permettaient le plus souvent pas de résoudre, était le caractère «neurogène » ou «myogène » de l'atteinte musculaire, un neurologue et pathologiste belge, Christian Coërs, entreprit d'étudier directement le dispositif d'innervation motrice en utilisant la méthode décrite par Ehrlich de coloration «vitale» par le Bleu de Méthylène, «se soustrayant » ainsi qu'il écrit lui-même, «à cette sorte de fascination que les procédés d'imprégnation métallique exerçaient depuis longtemps sur la plupart des histologistes qui étudiaient le système nerveux ». Coërs lui associa la visualisation des activités cholinestérasiques des plaques motrices par la technique de Koëlle qu'il vint apprendre chez Couteaux. Coërs, bientôt secondé par A.L. Woolf, développa une technique de repérage de la zone d'innervation par stimulation électrique au point moteur, et pratiqua in vivo des injections de bleu de méthylène, suivies d'une fixation par une solution à $10 \%$ de molybdate d'ammonium [4]. Ceci lui permit de faire un large inventaire des modifications du dispositif d'innervation terminal dans diverses conditions pathologiques, de déterminer un «Rapport d'innervation terminal» (rapport entre le nombre d'axones terminaux et celui des fibres musculaires innervées - ou nombre de fibres innervées par un axone terminal), rapport qui reste voisin de 1 dans les dystrophies et les atteintes «myogènes », et s'élève dans les atteintes neuropathiques. L'analyse des biopsies musculaires entrait dans une nouvelle ère, à la fois plus précise et d'une certaine façon quantifiable. Ce travail original fut poursuivi par D. Harriman en Angleterre et M. Fardeau en France à partir de 1958. Les variations de taille et de forme des plaques motrices dans diverses conditions pathologiques furent mises en évidence [5] et poursuivies à partir de 1962 par des études en microscopie électronique. De fait, ces études constituaient un premier pas vers une nouvelle analyse des biopsies musculaires.

\section{L'ère moderne}

L'application de techniques biochimiques à la visualisation de substrats ou d'activités enzymatiques sur coupes à congélation se développait en effet à la même époque en plusieurs endroits du monde (G. Gomori à Chicago, A.G.E Pearse à Londres, T. Takeuchi à Tokyo). Les applications au tissu musculaire squelettique se révélèrent immédiatement de premier intérêt, car permettant d'individualiser clairement différents types de fibres musculaires : d'abord à l'aide de réactions mettant en œuvre des activités oxydatives et phosphorylasiques (Ogata à Tokyo, V. Dubowitz dans le laboratoire de Pearse à Londres [6]), puis de techniques visualisant les activités ATP-asiques de la myosine (H. Padykula à Boston, L. Guth à Bethesda). Ces techniques prirent cependant leur véritable essor dans le laboratoire de Milton Shy au NIH (Bethesda) grâce aux travaux de W. King Engel. La netteté des résultats obtenus pour caractériser les différents types de fibres musculaires à l'aide de techniques de plus en plus simples et fiables, fut une vraie révolution dans l'analyse des biopsies musculaires. L'article publié en 1962 dans Neurology par W. King Engel [7] fut pendant des années l'article le plus référencé de la revue. Le laboratoire du NIH devint rapidement le lieu de convergence de tous les pathologistes et chercheurs travaillant sur les tissus musculaires. 


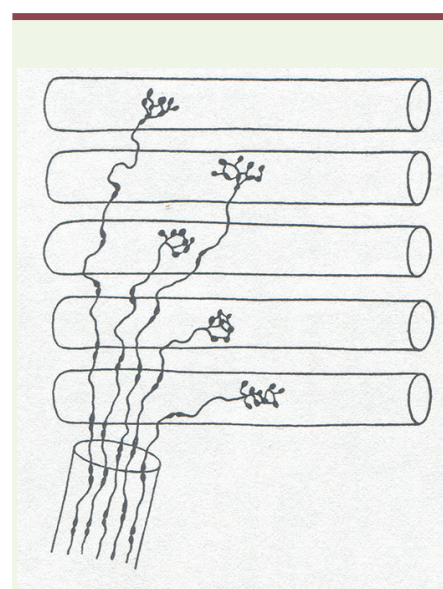

I. Normal arrangement of terminal innervation. Note one to one relationship, i.e. one nerve fibre, one end plate, one muscle fibre. T.I.R. $=1$,

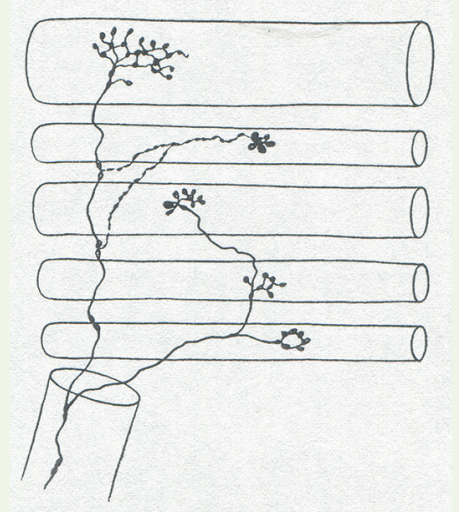

4. Reinnervation is now fully established. One nerve fibre innervates five muscle fibres. T.I.R. $=5$. Note change in innervation pattern and increased variation in form of end plates. Sprouts arising from near two nodes of Ranvier have joined.

Des perfectionnements et simplifications techniques pour visualiser sur le muscle adulte les trois types principaux de fibre musculaire furent mises au point dans ce laboratoire, en particulier par M.H. Brooke [8]. Une corrélation précise et rigoureuse avec les données électrophysiologiques, grâce à la technique de déplétion glycogénique provoquée par les stimulations nerveuses, fut établie avec la collaboration de W. Burke - toujours au NIH - révélant que les fibres d'une même unité motrice étaient toutes du même type. On pouvait donc pour la première fois « voir » sur une biopsie le territoire des unités motrices. Les applications aux biopsies provenant de pathologies neuromusculaires variées se révélèrent immédiatement si fructueuses que la généralisation de ces méthodes fut réellement explosive, permettant de révéler des champs pathologiques entièrement nouveaux: myopathies congénitales structurelles, dans la lignée de la «Central Core Disease» identifiée par Shy et Magee, «nemaline myopathies » myopathies centronucléaires, etc., mais aussi myopathies métaboliques, avec surcharge glycogénique ou lipidique et myopathies mitochondriales. Les corrélations avec les données de la microscopie électronique suivirent très rapidement: la pathologie musculaire «moderne » était née.

Dès lors, les techniques d'analyse en microscopie optique des biopsies musculaires furent rapidement standardisées : une «batterie » d'une vingtaine de techniques histologiques, cytochimiques et cytoenzymologiques sur coupes sériées à congélation faites au cryostat, formant le « cœur » de ces techniques. Elles furent après quelques années complétées par la mise au point de techniques immunocytochimiques à l'aide d'anticorps dirigés contre le système majeur d'immunocompatibilité, puis contre les myosines, les protéines membranaires, etc. L'analyse en microscopie électronique des biopsies s'articulait avec celles-ci afin d'identifier la nature et l'origine des anomalies décrites, et de détecter les inclusions non décelées ou difficiles à identifier en microscopie optique, comme les anomalies de structure mitochondriale, les agrégats tubulaires, les diverses inclusions intra-cytoplasmiques ou intranucléaires, etc. Les techniques histologiques classiques, après fixation

formolée et inclusion en paraffine étaient dès lors limitées à la recherche d'anomalies vasculaires ou inflammatoires interstitielles (granulomes sarcoïdiens par exemple) et à l'analyse des parasitoses ou des tumeurs. Le conditionnement des fragments prélevés par biopsies évoluait en conséquence dans ses modalités et dans la nécessaire rigueur des manipulations: congélation immédiate dans l'isopentane refroidi à $-180^{\circ}$ par l'azote liquide, fixation aldéhydique pour les fragments destinés à la microscopie électronique, la fixation formolée restant utilisée uniquement pour les fragments destinés à l'inclusion en paraffine et à leur étude en histologie classique. En fonction des questions pathologiques posées, des prélèvements complémentaires peuvent être nécessaires pour les analyses biochimiques particulières et conservés dans l'azote liquide, ou à $-80^{\circ}$, ou pour des cultures cellulaires. Pour les études d'innervation terminale et des plaques motrices, les prélèvements requièrent repérage per-opératoire. Ces techniques particulières seront précisées dans un article ultérieur.

\section{Quant aux techniques de biopsie elles-mêmes, elles ont évolué différemment selon les traditions « culturelles 》 des différentes écoles}

Un grand nombre de laboratoires, sans doute une majorité, ont développé les techniques de prélèvement à ciel ouvert (open biopsies) : aux États-Unis, au Canada, en France, en Italie, en Allemagne... Les interventions s'effectuent tantôt en milieu chirurgical, sous anesthésie locale ou générale selon l'âge des patients et les conditions hospitalières, avec présence dans la salle d'opération du technicien/ ou du médecin chargé du recueil et de la préservation immédiate des prélèvements; tantôt dans de petites salles dédiées à la chirurgie ambulatoire et à la pratique de biopsies, celles-ci étant effectuées par des médecins cliniciens et/ou pathologistes spécialement formés, assistés de techniciens(nes) en charge du recueil des prélèvements. Les modalités pratiques varient éventuellement en fonction des laboratoires aux fins d'obtenir les meilleurs résultats possibles en microscopie optique et électronique. À l'hôpital de La Pitié-Salpêtrière, des techniques particulières ont été développées dès les années 1960 en fonction de contextes cliniques et pathologiques particuliers: biopsies «couplées » nerf périphérique - tissu musculaire et «mini-open biopsies » chez les tout-petits.

- Chez les patients présentant une atrophie musculaire distale associée à une atteinte nerveuse périphérique, par exemple dans les amyotrophies Charcot-Marie, il fut proposé de réaliser des biopsies couplées, en un temps, de tissu musculaire et de nerf périphérique. La proximité ana- 
tomique, très favorable, du muscle court péronier latéral et de la branche terminale, purement sensitive, du nerf musculo-cutané, fut à l'origine de cette proposition de technique opératoire. Cette technique sera précisée dans le chapitre «Matériel et Méthodes » d'un travail consacré à l'étude des amyotrophies de type «Dejerine-Sottas [9]. Elle sera montrée aux différents pathologistes intéressés par ces affections, et reprise et publiée quelques années plus tard par l'équipe de la Mayo Clinic.

- Ce furent ensuite les «mini-open biopsies» chez les tout-petits : Ia fréquence de plus en plus grande des demandes concernant les hypotonies sévères du nourrisson, et l'importance du diagnostic pathologique dans ces conditions, firent se développer une technique de biopsie utilisant une instrumentation dérivée de la chirurgie plastique et ophtalmologique (utilisation par exemple d'écarteurs à paupière pour maintenir ouvertes les lèvres de l'incision cutanée). Ceci permettait de réaliser sur le chef moyen du deltoïde du petit enfant l'ensemble des prélèvements nécessaires au diagnostic par une incision de six à huit millimètres de longueur, dans d'excellentes conditions de visibilité et de sécurité. Cette technique fut très utilisée par M. Fardeau à partir des années 1970.

- Certains centres ont au contraire opté pour le développement des techniques de ponction-biopsie, en particulier en Angleterre et en Scandinavie. Différents types de trocarts ont été mis au point et utilisés de façon routinière [10-12]. Les ponctions-biopsies ont été utilisées essentiellement chez l'enfant et dans les études nécessitant des prélèvements itératifs par exemple chez les sportifs et pour l'étude de la sarcopénie et du vieillissement musculaire. Les prélèvements réalisés permettent toutes les analyses cytochimiques, cytoenzymologiques et biochimiques; ils sont moins favorables pour les études ultrastructurales et ne permettent pas les études du dispositif d'innervation. Dans la plupart des laboratoires pratiquant des biopsies « à ciel ouvert », les ponctions-biopsies sont généralement réservées aux analyses faites secondairement aux fins d'études biochimiques complémentaires ou de mises en culture cellulaires.

- Avec les progrès réalisés ces dernières années en imagerie musculaire, on peut aujourd'hui proposer de réaliser des prélèvements, en dehors des sites classiques de biopsie, dans des territoires identifiés comme importants pour le diagnostic. Ceci est en particulier utile pour certaines atteintes inflammatoires ou pour des atteintes focales du tissu musculaire.

\section{Conclusion}

Cette évolution des techniques de prélèvement souligne l'importance de la formation des opérateurs. II est bon qu'ils aient au moins une initiation aux gestes chirurgicaux (à cet égard la chirurgie expérimentale est une excellente préparation). La formation, ensuite, par une personne entraînée est indispensable. Il est essentiel également que les opérateurs connaissent la suite des opérations sur les prélèvements jusqu'à leur examen au microscope, afin de bien connaître les difficultés d'interprétation qu'entraînent les pincements, étirements, dilacérations, ou taille trop petite des prélèvements effectués. Inutile de préciser que l'emploi du bistouri électrique est rédhibitoire.

Cette évolution des techniques et leur diffusion ont nécessité une évolution juridique et administrative de ses prescriptions. Autre-

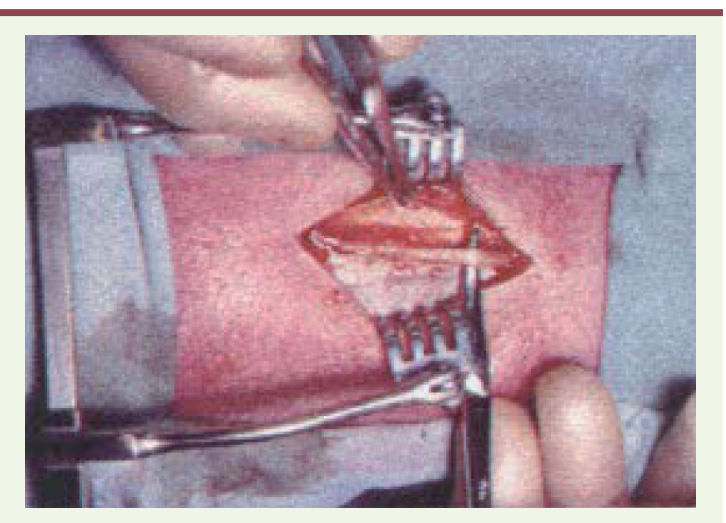

Photographie per-opératoire d'une biopsie couplée court péronier latéral et nerf musculo-cutané.

fois précédé d'un simple consentement oral à sa prescription, tout prélèvement biopsique nécessite aujourd'hui de façon impérative, après information complète du patient, ou des parents s'il s'agit d'un enfant, un consentement écrit, signé et daté, qui doit être conservé dans les cahiers de comptes rendus opératoires. $\diamond$

About the technique of muscle biopsy - A historical flash-back on the technique of muscle biopsie

\section{LIENS D'INTÉRÊT}

L'auteur déclare n'avoir aucun lien d'intérêt concernant les données publiées dans cet article.

\section{RÉFÉRENCES}

1. Duchenne GBA (1883). L'Électricisation localisée, $3^{\text {rd }}$ ed. $1872: 486-563$. Translated by Poore GV. Selections from the clinical works of Dr Duchenne (de Boulogne). London : The New Sydenham Society, pp 42-87.

2. Adams RD, Denny Brown D, Pearson CM. Diseases of muscle. A study in Pathology, $2^{e}$ ed. London : Henry Kimpton Limited, 1962.

3. Taxi J. René Couteaux ou la vision moderne de la jonction neuromusculaire. Les Cahiers de Myologie 2011 ; 4 : 5-9.

4. Coërs C, Woolf AL. The innervation of muscle. A biopsy study. Oxford: Blackwell, 1959.

5. Fardeau M. Technic and results of the study of motor plates in the pathology of skeletal muscle. Rev Neurol (Paris) $1960 ; 103: 30-40$.

6. Dubowitz V, Pearse AGE. Enzymic activity of normal and diseased human muscle: a histochemical study. J Pathol Bacteriol 1961; 81 : 365-78.

7. Engel WK. The essentiality of histo- and cytochemical studies of skeletal muscle in the investigation of neuromuscular disease. Neurology $1962 ; 12$ 778-94 (commentary : Neurology $1998 ; 51: 655$ ).

8. Brooke MH, Kaiser KK. Muscle fiber types: how many and what kind? Arch Neurol $1970 ; 23: 369-79$.

9. Garcin R, Lapresle J, Fardeau M, De Recondo J. Electron microscopic study of a peripheral nerve biopsy specimen in four cases of Dejerine-Sottas hypertrophic neuritis. Rev Neurol 1966 ; 115 : 917-32.

10. Bergström J. Muscle electrolytes in man. Scand J Clin Lab Invest $1962 ; 14$ (suppl) : 1-110.

11. Edwards R. Percutaneous needle-biopsy of skeletal muscle in diagnosis and research. Lancet 1971 ; ii : 593-5.

12. Henriksson KG. Semi-open muscle biopsy technique. A simple outpatient procedure. Acta Neurol Scand 1979 ; 59 : 317-23.

\section{TIRÉS À PART}

M. Fardeau 